\title{
Epidemiologia da Doença de Chagas Aguda no Nordeste Brasileiro
}

\author{
Epidemiology of Acute Chagas Disease in Northeast Brazil \\ Epidemiología de la enfermedad de Chagas aguda en el noreste de Brasil
}

Recebido: 18/04/2021 | Revisado: 23/04/2021 | Aceito: 08/05/2021 | Publicado: 22/05/2021

\author{
Silmara Ferreira de Oliveira \\ ORCID: https://orcid.org/0000-0001-8863-5075 \\ Centro Universitário Uninovafapi, Brasil \\ E-mail: aramlis.o@hotmail.com \\ Ana Paula Leal Lisboa \\ ORCID: https://orcid.org/0000-0002-6415-1110 \\ Centro Universitário Unifacid, Brasil \\ E-mail: anapaulaleallisboa7@ hotmail.com \\ Ariela Karollyny Santos Silva \\ ORCID: https://orcid.org/0000-0002-4489-5593 \\ Centro Universitário Uninovafapi, Brasil \\ E-mail: arialbuquerque1@ hotmail.com \\ Odilea Ribeiro Sanção \\ ORCID: https://orcid.org/0000-0003-0099-9531 \\ Centro Universitário Uninovafapi, Brasil \\ E-mail: odilea_ribeiro@hotmail.com \\ Augusto César Evelin Rodrigues \\ ORCID: https://orcid.org/0000-0001-7469-981X \\ Centro Universitário Unifacid, Brasil \\ E-mail: augustocevelin@yahoo.com.br
}

\begin{abstract}
Resumo
Objetivo: Determinar o perfil epidemiológico dos pacientes com doença de Chagas aguda (DCA) na região Nordeste do Brasil. Metodologia: Estudo epidemiológico descritivo, com abordagem quantitativa, baseado em dados secundários coletados no Sistema de Informação de Agravos de Notificação (SINAN) referentes aos casos de DCA notificados nos estados do Nordeste brasileiro, entre 2010 e 2019. Foram incluídos todos os casos notificados e registrados na base de dados do SINAN, excluindo-se aqueles que não se enquadravam no período delimitado para este estudo. A análise dos resultados foi feita através de números absolutos e relativos com resultados apresentados por meio de tabelas e gráficos. Resultados: O Nordeste é a segunda região mais afetada pelo DCA no Brasil, com predominância de casos no estado do Maranhão. O perfil sociodemográfico dos pacientes é, em sua maioria, composto por pessoas adultas, pardas e do sexo feminino. A DCA tem possível forma de transmissão principalmente por via oral e sem predomínio de zona de residência. Além disso, os dados de escolaridade e o local de infecção foram prejudicados, pois a informação foi ignorada em grande parte dos casos notificados. Conclusão: O estudo permitiu conhecer o perfil epidemiológico mais prevalente da DCA, com enfoque no estado do maranhão e evidenciando a forma de transmissão por via oral. Faz-se necessárias intervenções a partir de políticas de melhoria habitacional e combate ao vetor, investigação das contaminações orais, e estratégias de tratamento que visem melhorar a qualidade de vida dos infectados.
\end{abstract}

Palavras-chave: Mal de Chagas; Trypanosoma cruzi; Parasitologia.

\begin{abstract}
Objective: To determine the epidemiological profile of patients with acute Chagas disease (DCA) in the Northeast region of Brazil. Methodology: A descriptive epidemiological study, with a quantitative approach, based on secondary data collected in the Notifiable Diseases Information System (SINAN) referring to cases of ACD reported in the states of Northeast Brazil, between 2010 and 2019. All reported cases and registered in the SINAN database, excluding those that did not fit the period defined for this study. The analysis of the results was made through absolute and relative numbers with results presented through tables and graphs. Results: The Northeast is the second region most affected by DCA in Brazil, with a predominance of cases in the state of Maranhão. The sociodemographic profile of patients is mostly composed of adults, browns and females. DCA has a possible form of transmission, mainly orally and without a predominance of the area of residence. In addition, schooling data and the site of infection were impaired, as the information was ignored in most of the reported cases. Conclusion: The study allowed to know the most prevalent epidemiological profile of CAD, focusing on the state of Maranhão and showing the form of oral transmission. Interventions are needed based on policies to improve housing and combat the vector, investigation of oral contamination, and treatment strategies aimed at improving the quality of life of those infected.
\end{abstract}

Keywords: Chagas Disease; Trypanosoma cruzi; Parasitology. 


\begin{abstract}
Resumen
Objetivo: Determinar el perfil epidemiológico de pacientes con enfermedad de Chagas aguda (DCA) en la región Nordeste de Brasil. Metodología: Estudio epidemiológico descriptivo, con enfoque cuantitativo, basado en datos secundarios recolectados en el Sistema de Información de Enfermedades Notificables (SINAN) referentes a casos de DCA notificados en los estados del Nordeste de Brasil, entre 2010 y 2019. Se incluyeron todos los casos notificados y registrados en la base de datos del SINAN, excluyendo aquellos que no se ajustan al período definido para este estudio. El análisis de los resultados se realizó mediante números absolutos y relativos con resultados presentados a través de tablas y gráficos. Resultados: El Nordeste es la segunda región más afectada por DCA en Brasil, con predominio de casos en el estado de Maranhão. El perfil sociodemográfico de los pacientes está, en su mayor parte, compuesto por adultos, marrones y mujeres. El DCA tiene una posible forma de transmisión, principalmente por vía oral y sin predominio del área de residencia. Además, los datos de escolaridad y el sitio de la infección se vieron afectados, ya que la información se ignoró en la mayoría de los casos notificados. Conclusión: El estudio permitió conocer el perfil epidemiológico más prevalente de la EAC, centrándose en el estado de Maranhão y mostrando la forma de transmisión oral. Se necesitan intervenciones basadas en políticas para mejorar la vivienda y combatir el vector, investigación de la contaminación oral y estrategias de tratamiento dirigidas a mejorar la calidad de vida de los infectados.
\end{abstract}

Palabras clave: La enfermedad de Chagas; Trypanosoma cruzi; Parasitología.

\title{
1. Introdução
}

A doença de Chagas é causada pelo protozoário parasita Trypanosoma cruzi que é transmitido pelas fezes de um inseto (triatoma) conhecido como barbeiro. O nome do parasita foi dado por seu descobridor, o cientista Carlos Chagas, em homenagem ao também cientista Oswaldo Cruz. É uma patologia de caráter infeccioso, com fase aguda e crônica, sendo enquadrada dentro das enfermidades negligenciadas segundo a Organização Mundial de Saúde (Dias et al., 2016). As condições de pobreza e carência de opções terapêuticas eficazes e seguras estão intimamente relacionadas com tal fato, o que reforça a condição de endemia nos países do continente americano (Silva, Andrade Júnior \& Dantas, 2019).

No mundo, estima-se que 6 a 7 milhões de pessoas estejam infectadas com a DC, sobretudo na América Latina (Who, 2015). No Brasil, o cenário epidemiológico dessa doença mudou consideravelmente nos últimos 10 anos, como consequência das ações de controle, das transformações ambientais, sociais e econômicas. Atualmente, estima-se que 1,0 a 2,4\% dos brasileiros estejam infectados pela DC, o que corresponde a aproximadamente 1,9 a 4,6 milhões de pessoas (Dias et al., 2016).

A DC é uma antropozoonose causada pelo protozoário flagelado Trypanosoma cruzi, que tem como principais vetores, insetos triatomíneos hematófagos. A transmissão pode acontecer de várias formas, seja através do contato de solução de continuidade da pele ou mucosas com as fezes ou urina do inseto, ou através do consumo de alimentos contaminados com parasitos do triatomíneo infectado, ou ainda pelas vias transplacentária e transfusional (Coura, 2015; Brasil, 2017; Pinheiro et al., 2017). As apresentações desta patologia podem ocorrer em três fases: aguda, crônica assintomática e crônica sintomática. Destas, a fase aguda apresenta as manifestações mais críticas, o que na ausência de diagnóstico rápido e efetivo inevitavelmente evolui para fase crônica (Dias et al., 2016).

A fase aguda da DC é, na grande maioria dos casos, assintomática ( $90 \%$ dos casos), sendo caracterizada por elevada parasitemia, febre, mal-estar e a presença do sinal de Romanã, mais conhecido como 'chagomas', que são lesões oriundas de edema de mucosa ou edema cutâneo no local da inoculação. Em crianças, tende a apresentar-se de forma mais agressiva levando a complicações como miocardite e meningoencefalite, envolvendo bastante morbimortalidade (Silva, Aviz \& Monteiro, 2020).

Por não apresentar manifestações importantes, a fase aguda da DC costuma passar despercebida, levando a uma diminuição da quantidade de casos notificados nesta fase. Consequência disso é a prevalência do diagnóstico na fase crônica, onde a doença já pode manifestar-se nas formas indeterminada, cardíaca, digestiva ou cardiodigestiva no adulto, levando a internações recorrentes, licenças e óbitos precoces, ocasionando um grande impacto socioeconômico a esses pacientes (Pinto et al., 2007; Santos, 2018). 
Apesar a da alta taxa de morbimortalidade da DC no Brasil, ainda não é possível estimar a grandiosidade dessa doença, levando-se em consideração os dados referentes à vigilância epidemiológica, tendo em vista que apenas os casos na fase aguda eram compulsoriamente notificados através do Sistema de Informação de Agravos de Notificação (SINAN), e destes, acredita-se que apenas 10 a 20\% eram de fato notificados (Dias, 2016). Apenas em meados de 2020 a DC crônica passou a ser incluída na Lista Nacional de Notificação Compulsória de doenças, agravos e eventos de saúde pública nos serviços de saúde em todo o território nacional.

A DC é um problema de saúde global, sendo uma das principais doenças negligenciadas no Brasil e uma das quatro maiores causas de morte por doenças parasitárias e infecciosas (Martins-Melo et al., 2014). Dessa forma, a identificação do perfil epidemiológico e a distribuição espacial da doença, são importantes para gerar informações voltadas para a vigilância de saúde, no sentido de criar medidas intervencionistas para prevenir a doença e para identificar a necessidade de distribuição de recursos para aumentar o diagnóstico ainda na fase aguda e evitar a subnotificação. Considerando a magnitude do problema que a DC representa no âmbito da saúde pública e social e a escassez na literatura de publicações recentes sobre essa temática na região Nordeste, justifica-se a necessidade de realização da presente pesquisa.

Assim, esta pesquisa objetivou avaliar as características epidemiológicas e o perfil dos casos notificados e confirmados da doença de Chagas aguda (DCA) na Região Nordeste, por meio da descrição de casos registrados no SINAN, no período de 2010 a 2019.

\section{Metodologia}

Trata-se de estudo descritivo, epidemiológico, documental com abordagem quantitativa, baseado em dados secundários referentes aos casos de DCA notificados nos estados do Nordeste brasileiro, entre o período 2010 a 2019. De acordo com Rouquayrol \& Silva (2018), os estudos epidemiológicos permitem demonstrar a distribuição dos determinantes das doenças ou condições relacionadas à saúde em populações especificadas, enquanto o cunho descritivo permite a descrição da análise de dados em um período temporal determinado. Já a abordagem quantitativa é utilizada em levantamento de dados matemáticos por meio da utilização de porcentagens (Pereira et al., 2018).

A coleta dos dados ocorreu no mês de janeiro de 2021, por meio eletrônico, a partir do SINAN, base de dados disponibilizada pelo Departamento de Informática do Sistema Único de Saúde (DATASUS), com atualização dos casos de DCA mais recente em setembro de 2020. Foram incluídos todos os casos de DCA notificados e registrados na base de dados que tivessem unidade da federação (UF) de residência nos estados da região nordeste, excluindo-se aqueles que não se enquadravam no período delimitado para este estudo. As variáveis analisadas foram: UF de residência, sexo, raça, faixa etária, escolaridade, zona de residência, modo e local provável de infecção, critério de confirmação e evolução clínica.

Após coleta, procedeu-se a tabulação e análise dos dados através de estatística descritiva simples (números absolutos e relativos e porcentagem na base 100) no programa Microsoft Office Excel e os resultados foram apresentados na forma de tabelas e gráficos para melhor compreensão. Em razão da utilização de dados existentes em um banco de dados de domínio público, não houve a necessidade de submissão ao Comitê de Ética em Pesquisa (CEP) em concordância com a resolução 466/2012 do Conselho Nacional de Saúde.

\section{Resultados e Discussão}

O estudo analisou a ocorrência de 113 casos de DCA nos nove estados que compõem a região nordeste do Brasil, no período de 2010 a 2019. A região estudada é a segunda mais afetada pela doença, atrás apenas da região Norte que detém a quase totalidade dos casos em todo o país. 
Oliveira e Silva (2007) retratam que os estados do Nordeste contribuíam com 65\% da transmissão da doença no cenário nacional, sendo T. brasiliensis e T. pseudomaculata as espécies de vetores triatomíneos mais frequentemente capturadas pelo Programa de Controle da Doença de Chagas. Essas duas espécies são consideradas de difícil controle no contexto epidemiológico da endemia chagásica no nordeste brasileiro, o que pode justificar os dados atuais encontrados nesta pesquisa.

Gráfico 1 - Casos confirmados de Doença de Chagas aguda notificados na região Nordeste, entre 2010 e 2019, distribuídos segundo UF de residência.

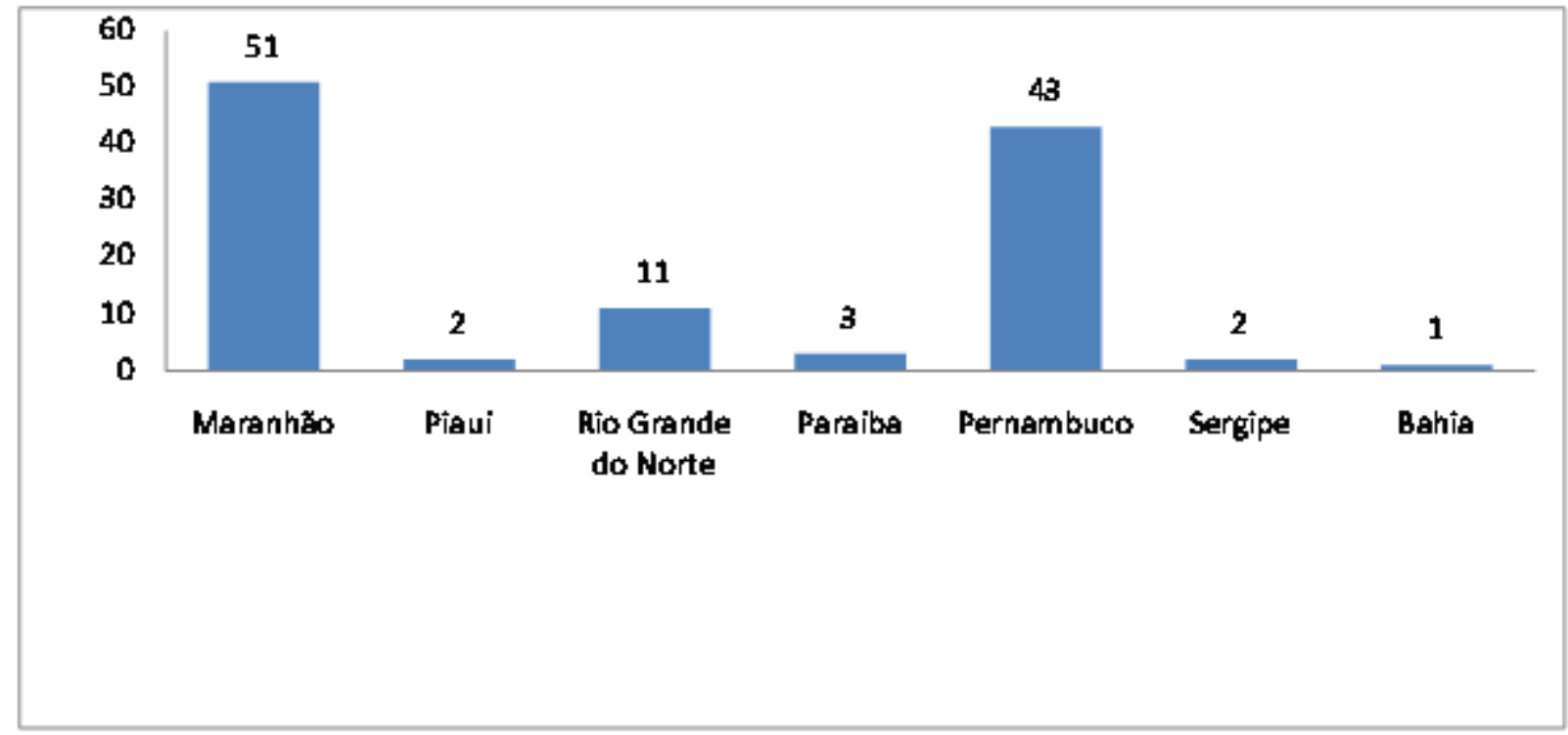

Fonte: Departamento de Informática do Sistema Único de Saúde (DATASUS) (2021).

O Gráfico 1 retrata a distribuição dos casos da região Nordeste entre os estados. Observa-se uma predominância entre os estados do Maranhão, com 51 casos, seguindo do Pernambuco, com 43 casos.

A literatura traz diversas pesquisas epidemiológicas direcionadas a esses estados, em especial o Pernambuco, onde mesmo após o controle do T. infestans, as espécies sinantrópicas T. brasiliensis, T. pseudomaculatae P. lutzi mantêm o risco de transmissão do T. cruzi ao homem no nesse estado. Estas espécies são amplamente distribuídas entre os municípios e espécimes infectados foram encontrados dentro das casas. Além disso, a presença marcante de P.lutziness e estado é importante, tendo em vista que essa espécie apresenta uma taxa de infecção natural superior às demais espécies analisadas (Silva et al., 2015).

Quanto ao estado do Maranhão, Simões Neto et al. (2018) relataram um surto epidêmico que acometeu 39 indivíduos em uma cidade no interior do estado. A via de transmissão implicada neste evento foi oral quando da ingesta de suco de bacaba (Oenocarpos bacaba). A DCA por transmissão oral se configura como importante forma de aquisição, podendo levar a graves consequências se não houver rápida suspeição. 
Tabela 1 - Distribuição dos casos de Doença de Chagas aguda notificados entre 2010 e 2019, de acordo com características sociodemográficas na Região Nordeste, Brasil.

\begin{tabular}{|c|c|c|}
\hline Variável & $\mathrm{N}=113$ & $\%$ \\
\hline \multicolumn{3}{|l|}{ Sexo } \\
\hline Masculino & 51 & $45 \%$ \\
\hline Feminino & 62 & $55 \%$ \\
\hline \multicolumn{3}{|l|}{ Raça/Cor } \\
\hline Branca & 30 & $26 \%$ \\
\hline Preta & 25 & $22 \%$ \\
\hline Amarela & 3 & $3 \%$ \\
\hline Parda & 53 & $47 \%$ \\
\hline Indígena & 1 & $1 \%$ \\
\hline Ignorado/Branco & 1 & $1 \%$ \\
\hline \multicolumn{3}{|l|}{ Faixa Etária (anos) } \\
\hline $1-4$ & 4 & $4 \%$ \\
\hline $5-9$ & 8 & $7 \%$ \\
\hline $10-14$ & 6 & $5 \%$ \\
\hline $15-19$ & 7 & $6 \%$ \\
\hline $20-39$ & 47 & $41 \%$ \\
\hline $40-59$ & 29 & $26 \%$ \\
\hline $60-64$ & 2 & $2 \%$ \\
\hline $65-69$ & 3 & $3 \%$ \\
\hline 70-79 & 5 & $4 \%$ \\
\hline $80 \mathrm{e}+$ & 2 & $2 \%$ \\
\hline \multicolumn{3}{|l|}{ Escolaridade } \\
\hline Nenhuma & 4 & $4 \%$ \\
\hline Ignorado/Branco & 109 & $96 \%$ \\
\hline \multicolumn{3}{|l|}{ Zona de Residência } \\
\hline Urbana & 54 & $48 \%$ \\
\hline Rural & 55 & $49 \%$ \\
\hline Ignorado/Branco & 4 & $3 \%$ \\
\hline
\end{tabular}

Fonte: Departamento de Informática do Sistema Único de Saúde (DATASUS) (2021).

De acordo com a Tabela 1, que retrata os aspectos sociodemográficos dos casos de DCA notificados, o sexo feminino prevaleceu com 55\% dos casos e a raça parda com 47\%. Quanto à faixa etária, predominaram as faixas de 20-39 anos com $41 \%$, e $40-59$ anos com $26 \%$. A escolaridade foi ignorada em $96 \%$ das notificações e a zona rural demonstrou uma leve prevalência com $49 \%$.

Observamos uma prevalência pouco acentuada do sexo feminino em relação ao masculino entre os casos. Essa variável não mostrou significativa diferença estatística, não podendo ser possível fazer relação risco para contaminação relacionada a ela. Andrade et al. (2020) também encontraram resultados semelhantes, demonstrando pouca relação de risco quando se fala em sexo em relação a esse agravo.

Quanto à raça, a cor parda predominou com $47 \%$ dos casos. Fato semelhante foi observado em estudos similares, onde se observaram prevalências da cor parda tanto em estudos nacionais como na região Norte (Andrade et al. 2020; Alencar et al., 2020). Além disso, a grande presença da população parda na região norte e nordeste do país mostra-se com um forte fator para a explicação dessa grande prevalência de acometimento em específico desta etnia (Brasil, 2021).

Ademais, é importante ressaltar que as desigualdades étnico-raciais em saúde no Brasil e em outros países possuem raízes em diferenças socioeconômicas, que se acumulam ao longo da vida de sucessivas gerações, levando a vivência em 
ambientes menos saudáveis, a assistência à saúde inadequada ou degradante além de experiências diretas de atos ou atitudes de discriminação, levando assim a efeitos próprios na saúde (Pearce et al., 2004).

A faixa etária predominante se concentrou entre 20 e 39 anos, com $41 \%$, seguido de 40 a 59 anos com 26\%. Esses dados são condizentes com outros estudos realizados em território brasileiro (Alencar et al., 2020; Bozelli et al., 2006; Cardoso et al., 2017; Pereira et al., 2017; Rodrigues et al., 2013; Silva, Andrade Júnior \& Dantas, 2019).

Isso sugere que possa existir relação entre atividade econômica, atividades laborais e os fatores de risco para transmissão da DCA. Esses dados são preocupantes, tendo em vista que estas faixas etárias são consideradas economicamente ativas e o seu adoecimento impacta negativamente no desenvolvimento das atividades devido comprometimento cardiovascular provocado pela doença, podendo resultar em mais absenteísmo e consequências socioeconômicas para o paciente e a família.

No que diz respeito à escolaridade, o maior número de casos (96\% do total) não foram especificados no momento da notificação ou foram ignorados, e apenas $4 \%$ dos casos com essa informação presente e com nenhuma escolaridade. Deve-se reforçar a importância para esta variável, assim como a demonstração do preenchimento prejudicado das fichas de notificação, sugerindo uma deficiência nos serviços de saúde. Ademais os casos que possuíam esta variável preenchida traziam como nenhuma escolaridade, demonstrando assim a vulnerabilidade social em que estão inseridos esses indivíduos.

Andrade et al. (2020), relatam que esses pacientes encontram-se envolvidos em uma situação econômica precária e isso faz com que morem em regiões periféricas, e lá estando, enfrentam a infraestrutura sanitária de péssima qualidade ou inexistente, com baixíssimos níveis de educação básica, sendo assim fator de risco que a expõe aos diversos tipos de transmissão da DC. Dessa forma, os estudos demonstram um expressivo número de pacientes chagásicos com baixa escolaridade, inclusive analfabetismo.

Em relação à zona de residência, não houve predomínio da zona urbana e rural, demonstrando que a doença está alterando seu perfil epidemiológico, que em tempos passados era restrita a ambientes silvestres, e atualmente evidencia um processo de urbanização da doença.

Atualmente existe no Brasil uma dinâmica de ocupação que implica a formação de novas ocupações urbanas e periurbanas, em localidades que são ambientalmente fragilizadas e com ocorrência da doença nessas localidades, seja por meio oral ou vetorial (Andrade et al. 2020).

Tabela 2 - Distribuição dos casos de Doença de Chagas aguda notificados entre 2010 e 2019, segundo unidade de federação e prováveis modo e local de infecção na Região Nordeste, Brasil.

\begin{tabular}{l|l|l|l|l|l|l|l|l}
\hline & MA & PI & RN & PB & PE & SE & BA & Total (N = 113) \\
\hline Modo Provável de Infecção & & & & & & & & \\
$\quad$ Vetorial & 5 & 1 & - & 1 & - & 1 & 1 & 9 \\
$\quad$ Oral & 41 & - & 9 & - & 29 & - & - & 79 \\
Ignorado/Branco & 5 & 1 & 2 & 2 & 14 & 1 & - & 25 \\
\hline Local Provável de Infecção & & & & & & & & \\
$\quad$ Domicílio & 28 & 1 & 1 & 1 & 2 & 2 & 1 & 36 \\
$\quad$ Outro & 11 & - & 6 & 1 & 12 & - & - & 30 \\
$\quad$ Ignorado/Branco & 12 & 1 & 4 & 1 & 29 & - & - & 47 \\
\hline
\end{tabular}

Fonte: Departamento de Informática do Sistema Único de Saúde (DATASUS) (2021).

Outra variável analisada foi relacionada o provável modo e local de infecção (Tabela 2). O maior número de casos, sendo 79 no total, está relacionado a forma oral como modo de transmissão, que pode estar diretamente relacionada ao consumo de tradicional de suco e polpa de frutas regionais contaminados, principalmente o açaí. Quanto ao local provável de 
infecção, a informação foi ignorada em grande parte das fichas de notificação, sendo as demais distribuídas sem prevalência entre domicílio e outros.

Tabela 3 - Distribuição dos casos de Doença de Chagas aguda notificados entre 2010 e 2019, de acordo com critério de confirmação e evolução na Região Nordeste, Brasil.

\begin{tabular}{l|l|l}
\hline Variável & $\mathbf{N}=\mathbf{1 1 3}$ & $\mathbf{\%}$ \\
\hline Critério de confirmação & & \\
$\quad$ Laboratorial & 104 & $92 \%$ \\
$\quad$ Clínico-epidemiológico & 4 & $3 \%$ \\
$\quad$ Ignorado/Branco & 5 & $5 \%$ \\
\hline Evolução & & \\
$\quad$ Vivo & 106 & $94 \%$ \\
$\quad$ Óbito pelo agravo & 1 & $1 \%$ \\
$\quad$ Ignorado/Branco & 6 & $5 \%$ \\
\hline
\end{tabular}

Fonte: Departamento de Informática do Sistema Único de Saúde (DATASUS) (2021).

Quanto ao critério de confirmação, $92 \%$ foram através de exames laboratoriais, com apenas 3\% de diagnóstico clínico-epidemiológico. Os demais foram categorizados como Ignorado/branco. De acordo com Andrade et al. (2020), os casos de DCA têm de ser confirmados através do diagnóstico laboratorial. No entanto, em algumas condições, adota-se o critério clínico epidemiológico para casos suspeitos e que estejam com os exames parasitológicos negativos e sorológicos inicialmente não reagentes e possam ter vínculo epidemiológico com outros casos anteriormente confirmados de DCA por critério laboratorial, durante surto de doença na fase aguda por transmissão oral (Andrade et al. 2020).

Finalmente, quanto a evolução clínica, 94\% dos pacientes tiveram boa evolução sendo enquadrados como vivos e apenas 1 progrediu para o óbito, sendo este caso no estado do Maranhão na cidade de Turiaço. Outros estudos têm demonstrado esse perfil, que apesar do alto risco de mortalidade da DC o desfecho clínico benéfico vem sendo observado e sinaliza um acesso satisfatório ao tratamento pela população acometida (Andrade et al. 2020).

\section{Considerações Finais}

Através deste estudo foi possível identificar o perfil epidemiológico e a distribuição espacial da doença entre os estados do Nordeste. Estes fatores são importantes na medida em que geram informações voltadas para a vigilância em saúde e prevenção da DCA, subsidiando a distribuição de recursos para aumento do diagnóstico ainda na fase aguda, evitando a subnotificação.

O perfil socioepidemiológico dos indivíduos acometidos pela doença foi: Pessoas economicamente ativas (adultas), pardas, sexo feminino, com possível forma de transmissão principalmente por via oral e sem predomínio de zona residência. Os dados de escolaridade e o local de infecção ficaram prejudicados, pois a informação foi ignorada em grande parte das fichas de notificação.

Sendo assim, percebe-se que os dados de notificação são importantes e tem grande potencial de delimitar o risco quando são feitos de maneira correta, por isso reforça-se a necessidade do preenchimento correto e completo da ficha de notificação.

Ademais, sugere-se que sejam considerados nas políticas de saúde os casos de transmissão oral, em especial no contexto do estado do Maranhão e Pernambuco aonde essa forma de transmissão vem ampliando sua relevância, além de subsidiar políticas que visem avanços na melhoria habitacional e no combate ao vetor. Embora tenha ocorrido redução da mortalidade chagásica no Brasil, a doença continua sendo um grave problema de saúde pública, principalmente na região 
Nordeste. Portanto, é fundamental que a assistência médica, a prevenção e o controle da doença de Chagas sejam mantidos e aprimorados.

\section{Referências}

Alencar, M. M. F., Santos Filho, R. A. B., Hirschheiter, C. A., Carmo, M. C. N., Santana, M. S. \& Ramos, J. L. D. et al. (2020). Epidemiologia da Doença de Chagas aguda no Brasil de 2007 a 2018. Research, Society and Development, 9(10), e8449109120. https://rsdjournal.org/index.php/rsd/article/view/9120/8278

Andrade, D.S., Teles, B. P., Lopes, D. I. S. \& Neves Neto, D. N. (2020) Análise do perfil epidemiológico dos pacientes acometidos por doença de chagas aguda notificados em Araguaína - TO no período de 2007 a 2018. Revista Cereus, 12(3), 212-227. http://ojs.unirg.edu.br/index.php/1/article/view/3217/1690

Batista, R. A. (2016). Doença de chagas aguda no estado do Maranhão: estudo do perfil cardiovascular. 39p. Monografia (Graduação em Medicina). Universidade Federal do Maranhão, São Luís. https://rosario.ufma.br/jspui/bitstream/123456789/2513/1/RodrigoAlmeidaBatista.pdf

Bozelli, C. E., Araújo, S. M., Guilherme, A. L. F. \& Gomes, M. L. (2006). Perfil clínico epidemiológico de pacientes com doença de Chagas no Hospital Universitário de Maringá, Paraná, Brasil. Cadernos de Saúde Pública, 22(5), 1027-1034. https://www.scielo.br/scielo.php?script=sci_arttext\&pid=S0102311 X2006000500015

Brasil. (2017). Guia de Vigilância em Saúde. Brasília: Ministério da Saúde, Secretaria de Vigilância em Saúde, Coordenação-Geral de Desenvolvimento da Epidemiologia em Serviços, 2017. https://portalarquivos.saude.gov.br/images/pdf/2017/outubro/06/Volume-Unico-2017.pdf

Brasil. (2021). Ministério da Saúde. Departamento de Informática do SUS. Informações de saúde (TABNET) - Epidemiológicas e Morbidade. http://tabnet.datasus.gov.br/cgi/tabcgi.exe?sinannet/cnv/chagasbr.def

Cardozo, E. J. S., Cavalcanti, M. A. F., Barreto, M. A. F. \& Nascimento, E. G. C. (2017). Perfil epidemiológico dos portadores de doenças de chagas: Dos indicadores de risco ao processo de enfrentamento da doença. Arquivos de Ciências da Saúde, 24(1), 41-46. https://www.cienciasdasaude.famerp.br/index.php/racs/article/view/545

Coura, J. R. (2015). The main sceneries of Chagas disease transmission. The vectors, bloodand oral transmissions - A comprehensive review. Memórias do Instituto Oswaldo Cruz, 110, 277-282. Retrieved from https://www.scielo.br/scielo.php?script=sci_arttext\&pid=S0074-02762015000300277

Dias, J. C. P., Ramos Jr., A. N., Gontijo, E. D., Luquetti, A., Shikanai-Yasuda, M. A. \& Coura, J. R. et al. (2016). II Consenso Brasileiro em Doença de Chagas, 2015. Epidemiologia e Serviços de Saúde, 25, 7-86. http://scielo.iec.gov.br/scielo.php?script=sci_arttext\&pid=S1679-49742016000500007

Martins-Melo, F. R., Lima, M. S., Ramos Jr, A. N., Alencar, C. H. M. \& Heukelbach, J. (2014). Revisão sistemática: Prevalência da doença de Chagas em gestantes e transmissão congênita do Trypanosoma cruzi no Brasil: Uma revisão sistemática e meta-análise. Tropical Medicine \&International Health, 19(8), 943-957. https://www.scielo.br/scielo.php?pid=S2237-96222016000500007\&script=sci_arttext\&tlng=pt

Oliveira, A. W. S. \& Silva, I. G. (2007). Distribuição geográfica e indicadores entomológicos de triatomíneos sinantrópicos capturados no Estado de Goiás. Rev Soc Bras Med Trop. v40: 204-208. https://www.scielo.br/scielo.php?pid=S0037-86822007000200011\&script=sci_abstract\&tlng=pt

Pearce, N., Foliaki, S., Sporle, A. \& Cunningham, C. (2004). Genetics, race, ethnicity, and health. BMJ: British Medical Journal, 328(7447), 1070-1072. https://www.ncbi.nlm.nih.gov/pmc/articles/PMC403856/

Pereira, A. S., Shitsuka, D. M., Parreira, F. J. \& Shitsuka, R. (2018). Metodologia Da Pesquisa Científica. UFSM. https://repositorio.ufsm.br/bitstream/handle/1/15824/Lic_Computacao_Metodologia-Pesquisa-Cientifica.pdf?sequence=1

Pereira, C. M. L., Azevedo, A. P., Marinho, S. S. B., Prince, K. A., Gonçalves, J. T. T. \& Costa, M. R. et al. (2017). Perfil clínico e epidemiológico da doença de chagas aguda no estado de Minas Gerais. Revista de Atenção à Saúde, 15(52), 49-54. https://seer.uscs.edu.br/index.php/revista_ciencias_saude/article/view/4523

Pinheiro, E., Brum-Soares, L., Reis, R. \& Cubides, J. (2017). Chagas disease: review of needs, neglect, and obstacles to treatment access in Latin America. Rev. Soc. Bras. Med. Trop., 50(3), 296-300, https://www.scielo.br/scielo.php?script=sci_arttext\&pid=S0037-86822017000300296

Pinto, A. Y. N., Farias, J. R., Marçal, A. S., Galúcio, A. L., Costi, R. R. \& Valente, V. C. et al. (2007). Doença de chagas aguda grave autócne da Amazônia brasileira. Revista Paraense de Medicina, 21(2). http://scielo.iec.gov.br/pdf/rpm/v21n2/v21n2a02.pdf

Rodrigues, J. R. A., Silva-Júnior, J. L. R., Paredes, A. O., Reis, A. S. \& Silva, L. A. C. (2013). Doença de Chagas Aguda no Estado do Maranhão, Brasil: Uma comparação entre os bancos de dados do SINAN e da FUNASA. Journal of Management \& Primary Health Care, 4(1), 3-9. https://www.jmphc.com.br/jmphc/article/view/160

Santos, F. R. (2018). Triagem neonatal para infecção da doença de chagas congênita: avaliação da prevalência ao nascer na região sul de Sergipe. 75. Dissertação (Mestrado em Ciências da Saúde). Universidade Federal de Sergipe, Aracaju-SE. Retrieved from https://ri.ufs.br/jspui/handle/riufs/7485

Silva, M. B. A., Menezes, K. R., Siqueira, A. M., Balbino, V. Q., Lorosa, E. S. \& Farias, M. C. G. et al. (2015). Importância da Distribuição Geográfica dos Vetores da Doença de Chagas em Pernambuco, Brasil, em 2012. Rev Patol Trop, vol. 44 (2): 195-206. https://www.revistas.ufg.br/iptsp/article/view/36650

Silva, G. G., Aviz, G. B. D. \& Monteiro, R. C. (2020). Perfil epidemiológico da Doença de Chagas aguda no Pará entre 2010 e 2017. Pará Research Medical Journal, 4, e29. https://www.prmjournal.org/article/10.4322/prmj.2019.029/pdf/prmjournal-4-e29.pdf

Silva, A. P. Da, Andrade Júnior, F. P. D. \& Dantas, B. B. (2019). Doença de Chagas: Perfil de morbidade hospitalar na Região do Nordeste Brasileiro. Revista de Ciências da Saúde Nova Esperança, 17(3), 08-17. https://revista.facene.com.br/index.php/revistane/article/view/550 
Research, Society and Development, v. 10, n. 6, e10310615190, 2021

(CC BY 4.0) | ISSN 2525-3409 | DOI: http://dx.doi.org/10.33448/rsd-v10i6.15190

Simões Neto, E. A., Costa, J. M. L., Simões, A. F., Sodré, D. C., Almeida, I. A. \& Costa, A. C. M. et al. (2018). Surto epidêmico de Doença de Chagas aguda (DCA) por transmissão oral no Maranhão: relato de 39 casos. In: Congresso Da Sociedade Da Sociedade Brasileira De Medicina Tropical, $54 ., 2018$, Olinda, Pernambuco: Sociedade Brasileira de Medicina Tropical. 1 p. pôster. https://www.arca.fiocruz.br/handle/icict/29855

Rouquayrol, M. Z. \& Silva, M. G. C. (2018). Epidemiologia \& saúde. Medbook.

Who. (2015). Chagas disease in Latin America: an epidemiological update basedon 2010 estimates = Maladie de Chagas en Amérique latine: le point épidémiologique base surles estimations de 2010. Weekly Epidemiological Record = Relevé épidémiologique hebdo madaire, 90 (06), 33 - 44. https://apps.who.int/iris/handle/10665/242316 